ARTICLE

https://doi.org/10.1038/s41467-022-28241-8

\title{
OPEN
}

\section{Internal Stark effect of single-molecule fluorescence}

Kirill Vasilev (1) ', Benjamin Doppagne1, Tomáš Neuman @i ', Anna Rosławska', Hervé Bulou', Alex Boeglin (1) 1, Fabrice Scheurer ${ }^{1} \&$ Guillaume Schull ${ }^{1 \times}{ }^{1 凶}$

The optical properties of chromophores can be efficiently tuned by electrostatic fields generated in their close environment, a phenomenon that plays a central role for the optimization of complex functions within living organisms where it is known as internal Stark effect (ISE). Here, we realised an ISE experiment at the lowest possible scale, by monitoring the Stark shift generated by charges confined within a single chromophore on its emission energy. To this end, a scanning tunneling microscope (STM) functioning at cryogenic temperatures is used to sequentially remove the two central protons of a free-base phthalocyanine chromophore deposited on a $\mathrm{NaCl}$-covered $\mathrm{Ag}(111)$ surface. STM-induced fluorescence measurements reveal spectral shifts that are associated to the electrostatic field generated by the internal charges remaining in the chromophores upon deprotonation.

\footnotetext{
${ }^{1}$ Université de Strasbourg, CNRS, IPCMS, UMR 7504, F-67000 Strasbourg, France. ${ }^{凶}$ email: schull@unistra.fr
} 
n many chemical and biological systems, the electric fields generated by embedded electrostatic charges regulate the absorption or emission energies of chromophores. ${ }^{1-3}$. This phenomenon, known as internal Stark effect (ISE), ${ }^{3-5}$ contrasts with Stark shifts induced by external electric fields $s^{6,7}$. It is at play in natural light-harvesting complexes where the sensitive optical properties of chlorophyll ${ }^{8,9}$ and carotenoid ${ }^{2,10}$ are adjusted by local charges in surrounding proteins and neighboring compounds to enable energy funneling. Similarly, electrostatic interactions between retinal chromophores and neighboring charged groups are responsible for wavelength regulation of vision ${ }^{1}$. In these examples, ISE occurs in complex landscapes composed of a large number of interacting organic systems. Scaling down these effects to single-molecules would be a step towards understanding the intimate interaction between biological pigments and their electrostatic environment, but has been so far limited to the spectroscopy of molecules in frozen matrices ${ }^{11-14}$ and scanningtunneling microscopy (STM) experiments ${ }^{15-18}$ where external electric fields were used to shift the molecular states. The effect of an electric field generated by an elementary charge located within a chromophore on its optical properties remains up to now a Gedankenexperiment. Here, we use free-base phthalocyanine $\left(\mathrm{H}_{2} \mathrm{Pc}\right)$ molecules, deposited on a $\mathrm{NaCl}$-covered silver sample, as a model system to study the ISE induced by one or two charges localised at the center of the chromophore on its fluorescence properties. To do so, we successively remove the two central protons of a $\mathrm{H}_{2} \mathrm{Pc}$ molecule. STM images, topographic time traces, and differential conductance spectra are used to identify the nature of the deprotonated species and their electronic structure. STM-induced luminescence (STML) spectra recorded on the singly and doubly deprotonated compounds reveal a fluorescence emission blue-shifted compared to the original $\mathrm{H}_{2} \mathrm{Pc}$ chromophore. Based on a comparison with time-dependent density functional theory (TD-DFT) simulations, we show that these shifts can be traced back to the radial electric field generated by charges confined to the $\sigma$-orbitals of the deprotonated chromophores whereas their $\pi$-orbitals remain unchanged. As a consequence, the neutral and deprotonated compounds are isoelectronic, in contrast with scanning probe experiments in which charging a molecule alters its $\pi$-orbitals ${ }^{19-24}$.

The deprotonation procedure also affects the vibronic emission of the molecule, inducing measurable frequency shifts for several modes, an effect that is discussed in terms of a vibrational Stark effect $^{25}$ and mass changes similar to isotopic shifts. Overall, our experiment constitutes an ultimate ISE experiment where the electric field is generated directly inside the probed chromophore, a model landmark for more complex ISE-induced color-tuning phenomena occurring in biological systems, and a novel strategy to develop tunable optoelectronic devices relying on single molecules as active components.

\section{Results}

Deprotonation of $\mathrm{H}_{2} \mathrm{Pc}$ molecules on $\mathrm{NaCl} / \mathrm{Ag}(111)$. Figure 1a shows a sketch of the STM-induced luminescence (STML) experiment used to probe the Stark effect generated by central charges on the fluorescence of a phthalocyanine chromophore deposited on a $\mathrm{NaCl}$-covered $\mathrm{Ag}(111)$ surface (see "Methods" for details). To realise this scheme, we worked with $\mathrm{H}_{2} \mathrm{Pc}$ molecules whose typical STM images recorded at $V=-2.5 \mathrm{~V}$ and $V=0.5 \mathrm{~V}$ are displayed in Fig. 1b. While the left image reveals the characteristic four-fold symmetry pattern of the highest occupied molecular orbital (HOMO), the right one reflects the twofold symmetry pattern of the lowest unoccupied molecular orbital (LUMO) (see Supplementary Figs. 1-3 and Supplementary Table 1). This latter can adopt two configurations rotated by $90^{\circ}$ from each other in successive images 26,27 , a behavior that has been formerly assigned to tautomerization, i.e., the permutation of the central hydrogen atoms between two equivalent sites of the molecule. This phenomenon can be tracked in the variation of the tip-sample distance $(\Delta \mathrm{z})$ versus time (Fig. 1c) recorded at constant current where it appears as two-level fluctuations ${ }^{24,27-31}$. As a next step of the experiment, we located the tip on top of the center of the $\mathrm{H}_{2} \mathrm{Pc}$ molecule and applied a positive voltage ramp at a constant current of $10 \mathrm{pA}$ while simultaneously recording the relative tip-sample distance " $\mathrm{z}$ ". In most cases, this procedure reveals a sudden " $\mathrm{z}$ " decrease for $V \approx 3.2 \mathrm{~V}$ (see Supplementary Note 1 for details), hinting towards a change of the molecular structure. STM images recorded at $V=-1.5 \mathrm{~V}$ (HOMO) after such an event are similar to the one measured before, but are fuzzier (Fig. 1d). $\Delta z$ time-traces now reveal four-level fluctuations with a high switching frequency (Fig. 1e). This explains the fuzzy appearance of the HOMO image and hints towards the inner motion of a single proton in a HPc molecule $24,29,31$. This is confirmed by STM images acquired at $V=1.43 \mathrm{~V}$ [LUMO, (Fig. 1d)] where each tautomer can be stabilized and identified. The voltage is then ramped a second time until another sudden distance drop occurs. The typical four-fold symmetry STM images (Fig. 1f) observed for both HOMO and LUMO of the resulting compound ${ }^{32}$, together with non-fluctuating $\Delta z$ time traces (Fig. 1g) now suggest a doubly dehydrogenated (or deprotonated) phthalocyanine molecule (Pc).

Electronic structure of the deprotonated $\mathrm{H}_{2} \mathrm{Pc}$ molecules. Single and double STM-induced removal of central hydrogens of porphyrin and phthalocyanine molecules were reported in several prior works $24,29,31-33$. In some cases, it was assumed that only the proton was removed $29,31,33$ while some other works using partially decoupled molecules ${ }^{24,32}$ concluded on a full dehydrogenation (proton and electron). In the latter case, the HPc molecule is in a neutral state with an unpaired electron in the $\pi$ orbital, which strongly affects its electronic properties. The former, which has not been reported so far for decoupled molecules, should leave the molecule in a negatively charged state. Characterizing the charged state of the molecules may therefore unravel the exact nature of the STM-induced chemical reaction: dehydrogenation or deprotonation. On 2 monolayer (ML) NaClcovered (111) noble metal surfaces, charged atoms, and molecules were shown to scatter the two-dimensional surface-state localised at the metal-salt interface (also called interface state) while neutral species do $\operatorname{not}^{34,35}$. In Fig. 2a, e, and i, we display differential conductance images (i.e., constant current $\mathrm{d} I / \mathrm{d} V$ maps) recorded at a DC bias of $400 \mathrm{mV}$-some $200 \mathrm{meV}$ above the onset of the interface state-for the same molecule prior to (a) and after the first (e) and second (i) voltage ramp procedure (the topography is shown in the inset). These images reveal circular standing waves- which become even more apparent in difference images (see Supplementary Fig. 4)-around the modified molecules that are absent for $\mathrm{H}_{2} \mathrm{Pc}$. This indicates that, on $\mathrm{NaCl}$, only the protons are removed from the $\mathrm{H}_{2} \mathrm{Pc}$ molecules ending up in singly $\left(\mathrm{HPc}^{-}\right)$and doubly $\left(\mathrm{Pc}^{2-}\right)$ negative charged species. Note that the molecule occupies the same adsorption site prior to and after the deprotonation procedures (see Supplementary Fig. 5). In Fig. 2b, f and j, we first evaluate how these localised charges affect the electronic structure of the molecules by recording differential conductance $(\mathrm{d} I / \mathrm{d} V)$ spectra for the three compounds. These spectra reveal similar HOMO-LUMO gaps $(\approx 2.7 \mathrm{eV})$, as well as rigid shifts of the frontier orbitals to higher energies $\left(+0.94 \mathrm{eV}\right.$ for $\mathrm{HPc}^{-}$and $+1.05 \mathrm{eV}$ for $\left.\mathrm{Pc}^{2-}\right)$. For more information on the interface states and scattering of charged species, as well as on the rigidity of the electronic gap of the molecules, please refer to Supplementary Figs. 4-7. These observations further 


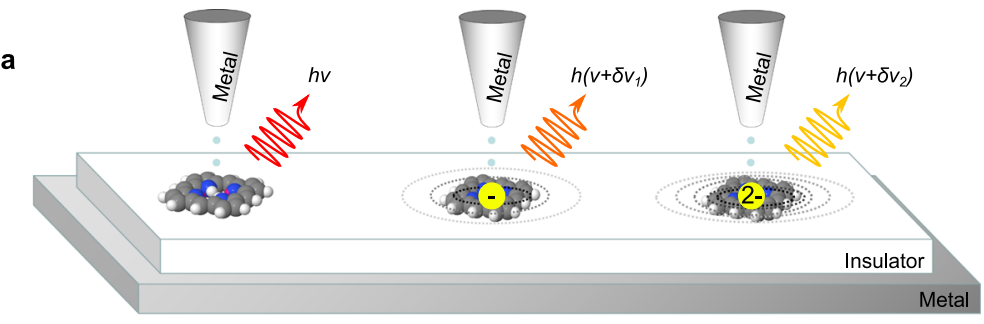

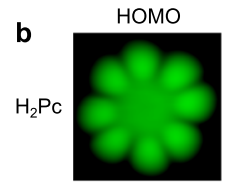

d
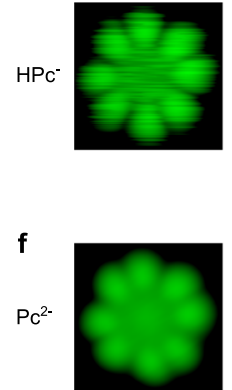
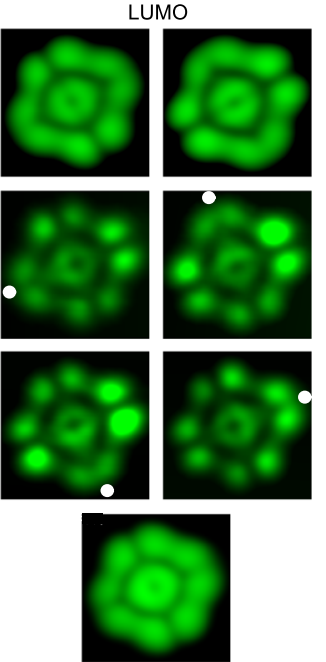

c
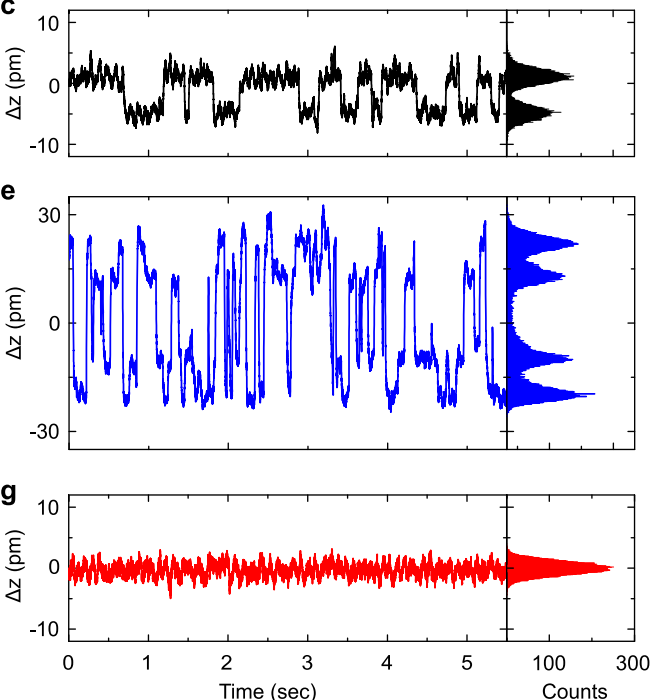

Fig. 1 Imaging protonated and deprotonated phthalocyanine molecules and monitoring tautomerization processes. a Sketch of the experiment where the fluorescence energy $(h \nu)$ of a phthalocyanine molecule is progressively Stark-shifted $\left(\delta h_{\mathrm{i}}\right)$ by charges located at the center of the chromophore. b, d, f STM images $\left(2.7 \times 2.7 \mathrm{~nm}^{2}\right)$ of a phthalocyanine molecule on $3 \mathrm{ML}-\mathrm{NaCl} / \mathrm{Ag}(111)$. The images of the HOMO were all recorded at $I=10 \mathrm{pA}$, with voltage $V=-2.5 \mathrm{~V}$ for $\mathrm{H}_{2} \mathrm{Pc}, V=-1.5 \mathrm{~V}$ for $\mathrm{HPc}^{-}$and $\mathrm{V}=-2 \mathrm{~V}$ for $\mathrm{Pc}^{2-}$. The $\mathrm{LUMO}$ were recorded at $I=10 \mathrm{pA}, \mathrm{V}=0.5 \mathrm{~V}$ for $(\mathbf{b}) \mathrm{H}_{2} \mathrm{Pc}, I=2.3 \mathrm{pA}$, $V=1.43 \mathrm{~V}$ for $(\mathbf{d}) \mathrm{HPc}^{-}$and $I=10 \mathrm{pA}, V=1.65 \mathrm{~V}$ for $(\mathbf{f}) \mathrm{Pc}^{2-}$. The LUMO images of $\mathrm{H}_{2} \mathrm{Pc}^{2}$ and $\mathrm{HPc}^{-}$reveal two and four different patterns, respectively, in successive images. The dots in the LUMO images of $\mathrm{HPc}^{-}$indicate the side where the remaining hydrogen atom is located. $\mathbf{c}, \mathbf{e}, \mathbf{g}$ Relative tip-sample distance $(\Delta z)$ time traces and their histograms recorded at a bias voltage of $-2.5 \mathrm{~V}$ on $(\mathbf{c}) \mathrm{H}_{2} \mathrm{Pc}_{1}(\mathbf{e}) \mathrm{HPc}^{-}$, and $(\mathbf{g}) \mathrm{Pc}^{2-}$, and at constant current ( $\mathrm{I}_{\text {set-point }}=10 \mathrm{pA}$ for $\mathrm{H}_{2} \mathrm{Pc}$ and $5 \mathrm{pA}$ for $\mathrm{HPc}^{-}$and $\mathrm{Pc}^{2-}$ ).

support the deprotonation mechanism, as a dehydrogenation would lead to a splitting of the HOMO into a singly occupied and a singly unoccupied molecular orbital ${ }^{24}$, or a shift of the original HOMO above the Fermi level ${ }^{32}$. The $d I / d V$ spectra in Fig. $2 b, f, j$ rather indicate that the $\pi$-orbitals are unaffected upon deprotonation, an observation that is backed-up by DFT calculations of the electronic structure of the three species (Fig. 2c, g, k), see Methods for details. In contrast, these calculations reveal large modifications of some $\sigma$ orbitals, originally involved in the $\mathrm{N}-\mathrm{H}$ bond. For example, the orbital labeled $\sigma$-HOMO in Fig. $2 \mathrm{~g}$ and $\mathrm{k}$, located $1.7 \mathrm{eV}$ below the $\pi$-HOMO in the calculation of $\mathrm{H}_{2} \mathrm{Pc}$, appears $0.3 \mathrm{eV}$ below and $0.4 \mathrm{eV}$ above the $\pi$-HOMO for $\mathrm{HPc}^{-}$and $\mathrm{Pc}^{2-}$, respectively. Altogether, these observations suggest that the excess negative charges do not localise in the frontier $\pi$-orbitals but in the $\sigma$-orbitals originally involved in the $\mathrm{N}-\mathrm{H}$ bonds. In fact, similar deprotonation effects were reported for porphycene compounds which were identified as $\sigma$-type anions and dianions ${ }^{36}$. To better identify where the excess charges are located on the $\mathrm{HPc}^{-}$and $\mathrm{Pc}^{2-}$ chromophores, we represent in Fig. $2 \mathrm{~d}, \mathrm{~h}$, and 1 the total electrostatic potential generated jointly by the nuclei and the distributed electron density of the singlet ground state of the molecule. The potential is displayed in a horizontal plane $0.21 \mathrm{~nm}$ above the molecule. The absence of clear contrast in the calculated image of $\mathrm{H}_{2} \mathrm{Pc}$ confirms the neutral nature of the molecule. For $\mathrm{HPc}^{-}$and $\mathrm{Pc}^{2-}$, these calculated images reveal an overall negative potential on the molecules, that is of maximum amplitude at the center of the chromophores where the protons have been removed. This indicates that the charges left over after deprotonation remain close to their original positions, strongly affecting the $\sigma$-orbitals originally involved in the $\mathrm{N}-\mathrm{H}$ bond but preserving the $\pi$-structure of the chromophore. The rigid shift of the frontier orbitals observed in Fig. $2 \mathrm{f}, \mathrm{j}$ can therefore be associated to a change in the molecular ionization energy due to the electric field generated by the excess inner charges. For more information about calculations of the electronic structure and total potential, please refer to "Methods".

Internal Stark-shift of the molecular fluorescence lines. We now discuss the photonic properties of the three chromophores and investigate the effect of one or two central charges on the fluorescence of the phthalocyanine molecule. Figure 3 displays the STM-induced fluorescence spectra of $\mathrm{H}_{2} \mathrm{Pc}, \mathrm{HPc}^{-}$and $\mathrm{Pc}^{2-}$. The spectrum of $\mathrm{H}_{2} \mathrm{Pc}$, excited at a negative bias of $V=-2.5 \mathrm{~V}$, is composed of two purely electronic contributions named $\mathrm{Q}_{x}$ and $\mathrm{Q}_{y}$ appearing respectively at $\approx 1.81$ and $\approx 1.93 \mathrm{eV}^{26,27,37,38} \cdot \mathrm{Q}_{x}$ $\left(\mathrm{Q}_{y}\right)$ is defined as the low (high) energy spectral contribution, which, for $\mathrm{H}_{2} \mathrm{Pc}$, is associated to a dipole oriented along (perpendicular to) the axis formed by the two inner hydrogen atoms. $\mathrm{Q}_{y}$ usually appears much weaker than the lower energy $\mathrm{Q}_{x}$ contribution, reflecting fast non-radiative decay channels between the excited states $^{39}$. Peaks of low intensities are also observed on the low energy side of the spectrum that reflect vibronic transitions characteristic of the $\mathrm{H}_{2} \mathrm{Pc}$ molecule 27,40 . Independently of the 

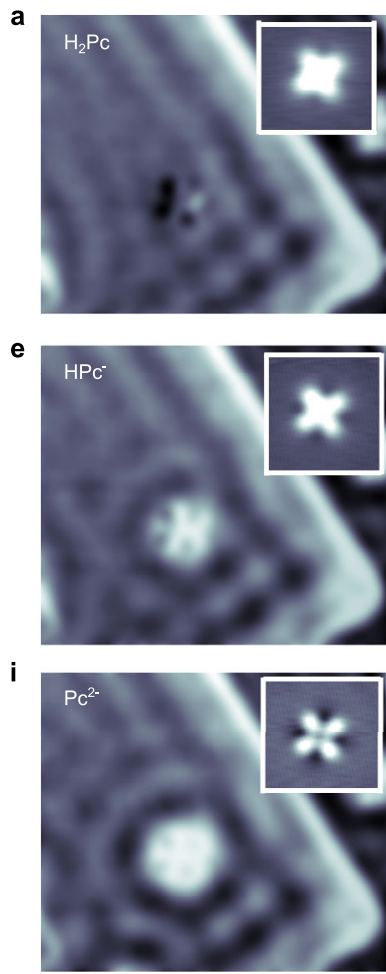
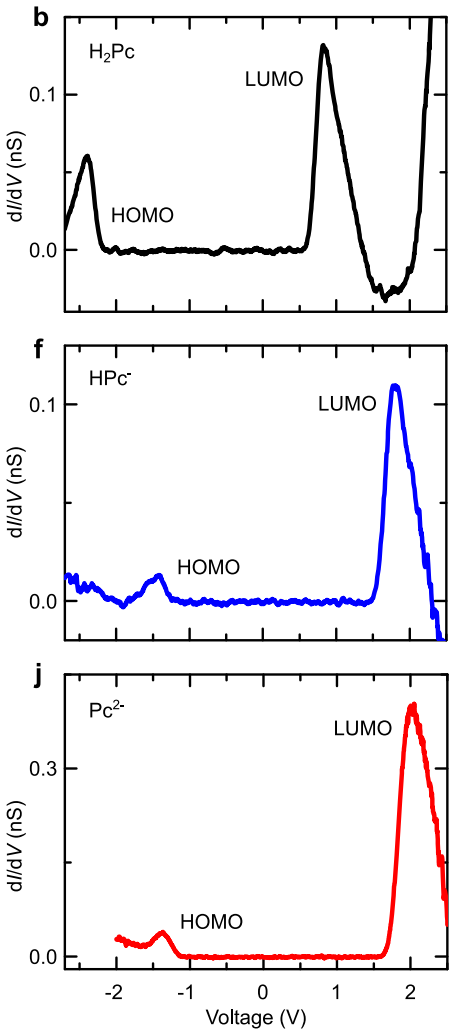
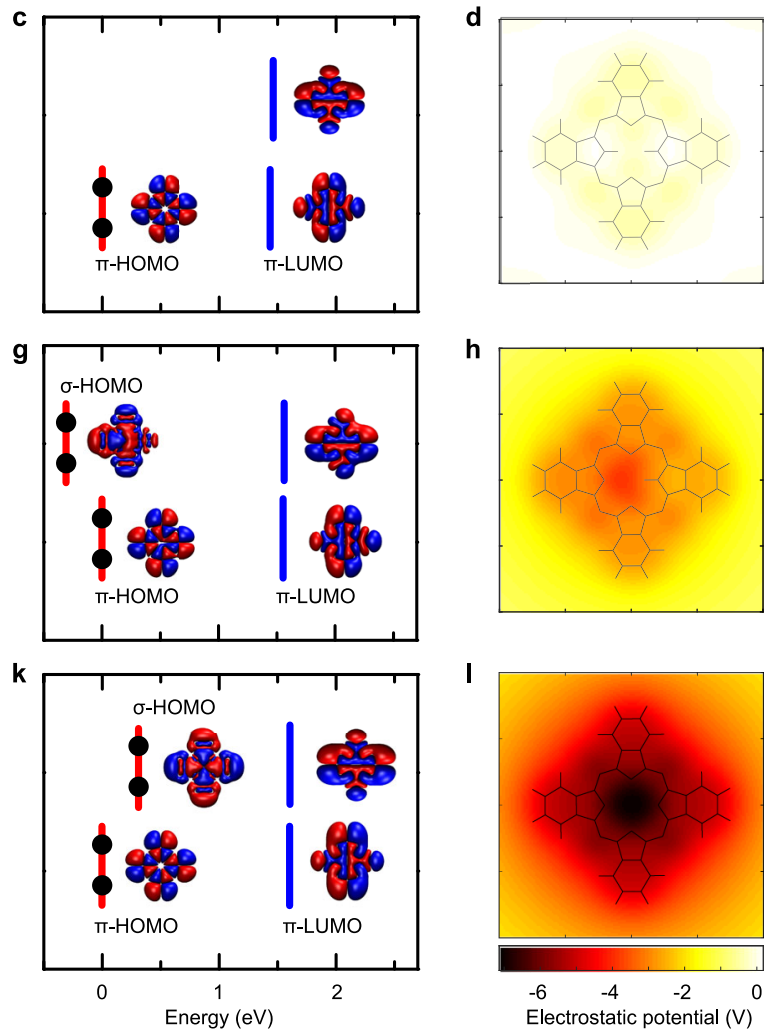

Fig. 2 Identifying charges confined in deprotonated phthalocyanines. a, e, i Constant current $\mathrm{d} / \mathrm{dV}$ maps $\left(I=30 \mathrm{pA}, V=400 \mathrm{mV}, 12.5 \times 10.8 \mathrm{~nm}{ }^{2}\right.$, modulation voltage $\delta V_{\text {mod }}=50 \mathrm{mV}$ ) recorded on $(\mathbf{a}) \mathrm{H}_{2} \mathrm{Pc}_{1}(\mathbf{e}) \mathrm{HPc}^{-}$, and (i) $\mathrm{Pc}^{2-}$. Images (a), (e), and (i) were acquired on a $2 \mathrm{ML}-\mathrm{NaCl}$ island where the interface state scattering is stronger than on $3 \mathrm{ML}$ for a better visualization of the scattering. The conclusions regarding the charged character of the different species on 2 or $3 \mathrm{ML} \mathrm{NaCl}$ remain however the same (see Supplementary Fig. 8). The insets show topographic STM images (3.7 $\times 3.7 \mathrm{~nm}{ }^{2}$ ) recorded simultaneously that reveal dark areas around $\mathrm{HPc}^{-}$and $\mathrm{Pc}^{2-}$, characteristic of charged molecules on $\mathrm{NaCl}{ }^{35} . \mathbf{b}, \mathbf{f}, \mathbf{j} \mathrm{d} / / \mathrm{dV}$ spectra recorded on $(\mathbf{b})$ $\mathrm{H}_{2} \mathrm{Pc}$, (f) $\mathrm{HPc}^{-}$, and (j) $\mathrm{Pc}^{2-}$ on $3 \mathrm{ML}$ of $\mathrm{NaCl}$. c, g, $\mathbf{k}$ DFT calculations of the electronic structure of the frontier orbitals of $(\mathbf{c}) \mathrm{H}_{2} \mathrm{Pc}_{1}(\mathbf{g}) \mathrm{HPc}^{-}$, and $(\mathbf{k}) \mathrm{Pc}^{2-}$ together with their iso-surface representations and their electronic occupation. $\mathbf{d}, \mathbf{h}, \mathbf{I}$ calculated images of the electrostatic field for $(\mathbf{d}) \mathrm{H}_{2} \mathrm{Pc}_{1}(\mathbf{h}) \mathrm{HPc}^{-}$, and (I) $\mathrm{Pc}^{2-}$. The potential is displayed $0.21 \mathrm{~nm}$ above the plane of the molecule. The $\mathrm{NaCl}$ layer is not included in the calculation. Source data are provided as a Source Data file.

used polarity, $\mathrm{HPc}^{-}$does not emit when directly excited by the STM tip, a behavior that will be discussed elsewhere but that we associate to the low absolute energies of both HOMO and LUMO that prevent the excitation of the molecule by tunneling electrons ${ }^{41}$. However, the fluorescence of $\mathrm{HPc}^{-}$can be recovered through excitonic energy transfer ${ }^{37,42}$ from a higher energy gap molecule, here $\mathrm{ZnPc}$, positioned in direct contact to the "dark" $\mathrm{HPc}^{-}$ (Supplementary Fig. 9). Hence, an energy-transfer mediated excitation enables probing the fluorescence of otherwise "dark" molecules, a strategy that may be used with other "dark" chromophores in STML experiments. This spectrum exhibits two electronic contributions; the high energy one can be associated to the $\mathrm{Q}_{Z n}$ emission line of the $\mathrm{ZnPc}$ donor ${ }^{40,43}$, whereas the $\mathrm{Q}_{x}$ fluorescence line of $\mathrm{HPc}^{-}$is at $\approx 1.86 \mathrm{eV}$, some $50 \mathrm{meV}$ above the $\mathrm{Q}_{x}$ peak of $\mathrm{H}_{2} \mathrm{Pc}$. Note that according to our TD-DFT calculations, the low energy transition $\left(\mathrm{Q}_{x}\right)$ of $\mathrm{HPc}^{-}$is oriented along the molecular axis that does not contain the hydrogen atom. The $\mathrm{Q}_{y}$ contribution of $\mathrm{HPc}^{-}$cannot be identified in the spectrum (Fig. 3b), probably because it is at a higher energy than the $\mathrm{Q}_{Z n}$ contribution of the $\mathrm{ZnPc}$ donor. For $\mathrm{Pc}^{2-}$, Fig. $3 \mathrm{c}$ shows that it can only be excited at positive voltage $(V=2.5 \mathrm{eV})$ with an emission line at $\approx 1.88 \mathrm{eV}$. Similar to metal phthalocyanines ${ }^{37,40,43,44}$, only a single emission line is observed in this spectrum, reflecting the D4h symmetry of the doubly deprotonated molecule and the associated degeneracy of the two first emission contributions.

In summary, one notices that moving from $\mathrm{H}_{2} \mathrm{Pc}_{\mathrm{c}}$ to $\mathrm{Pc}^{2-}$, the transition is blue-shifted by $50 \mathrm{meV}$ upon the first deprotonation, and by an additional $20 \mathrm{meV}$ upon the second one. These shifts may originate from several physical effects. Due to the presence of the STM tip generating external static and dynamic electrical fields, Stark and photonic Lamb effects may contribute to the observed energy shifts $18,45-48$. However, recent STML and photoluminescence works demonstrated that these effects may at best lead to few meV shifts of the emission maxima ${ }^{18,48}$, and cannot explain the large blue shifts reported in Fig. $3 \mathrm{~b}$ and c.

The interaction between the indirectly excited $\mathrm{HPc}^{-}$molecule with the donor molecule of $\mathrm{ZnPc}$ can also result in small energy shifts ${ }^{42}$ of the $\mathrm{HPc}^{-}$emission line, but which again are negligible compared to the shifts in Fig. 3. Similarly, we theoretically ruled out the impact of the static screening of the $\mathrm{NaCl}$ substrate on the energy shifts by performing DFT and TD-DFT calculations ${ }^{44}$ (discussed in detail in Supplementary Note 2). On the other hand, charged chromophores as $\pi$-type phthalocyanine anions and cations discussed in previous reports ${ }^{21-23,49}$ are systematically characterized by a strongly red-shifted emission $(\approx 400 \mathrm{meV})$ compared to neutral compounds, reflecting important modifications of the $\pi$-orbitals involved in the optical transition. Similarly, if one assumes neutral dehydrogenated compounds, one is left with one unpaired electron in the HOMO ( $\mathrm{HPc})$ or with an empty HOMO $(\mathrm{Pc})^{24,32}$. The fluorescence spectra of $\mathrm{HPc}$ and Pc should therefore reflect those of a $\mathrm{H}_{2} \mathrm{Pc} \pi$-type cation $\left(\mathrm{H}_{2} \mathrm{Pc}^{+}\right)$and a $\mathrm{H}_{2} \mathrm{Pc} \pi$-type dication $\left(\mathrm{H}_{2} \mathrm{Pc}^{2+}\right)$ with whom they share the same electronic structure. Fluorescence spectra of HPc and Pc should therefore display emission lines redshifted by roughly $400 \mathrm{meV}$ with respect to the $\mathrm{Q}_{x}$ of $\mathrm{H}_{2} \mathrm{Pc}$; this is 

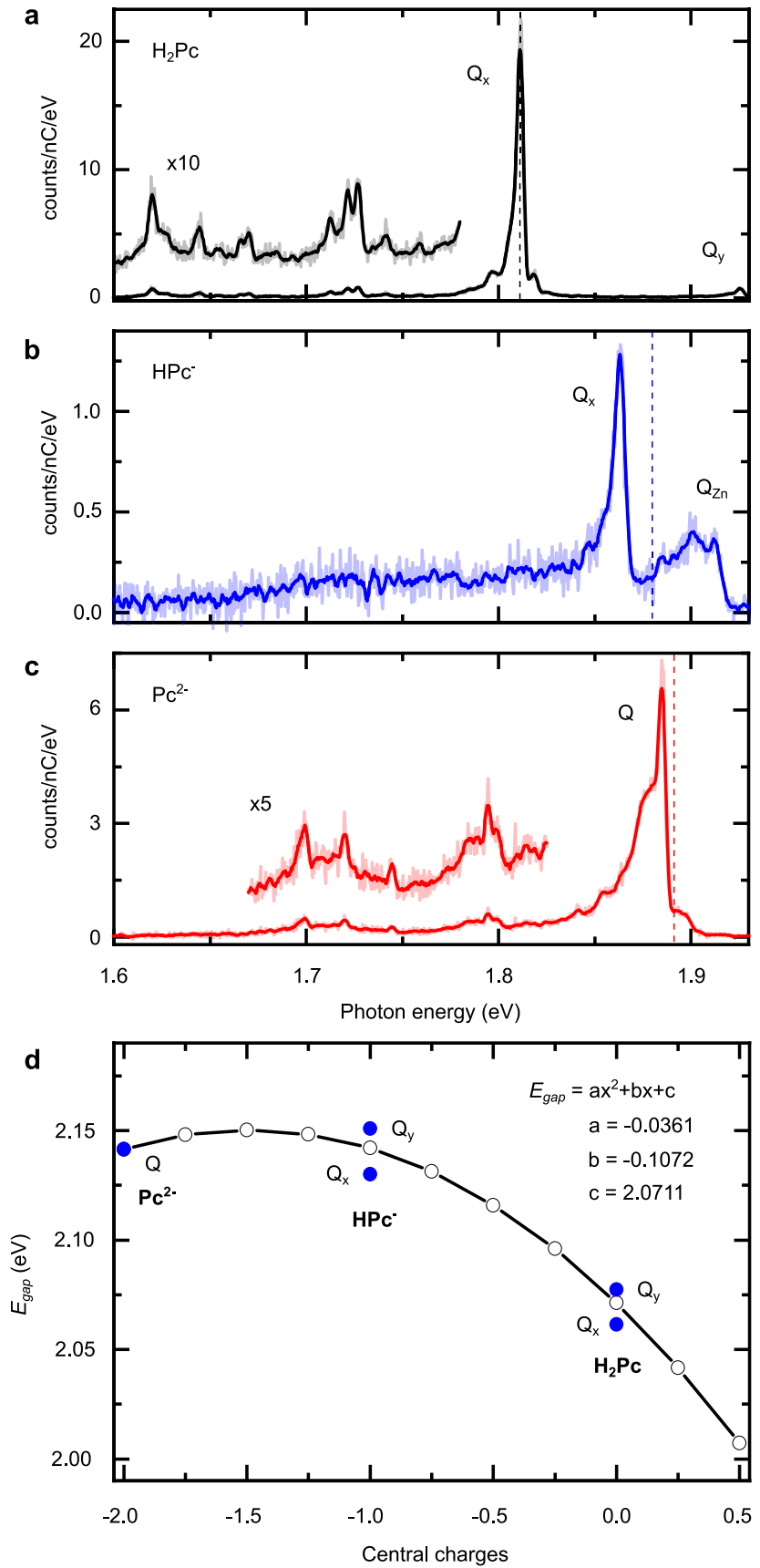

Fig. 3 ISE in fluorescence spectra of neutral and charged

phthalocyanines. STML spectra (acquisition time $t=120 \mathrm{~s}$ ) of (a) $\mathrm{H}_{2} \mathrm{Pc}$ $(I=100 \mathrm{pA}, V=-2.5 \mathrm{~V}),(\mathbf{b}) \mathrm{HPc}^{-}(I=60 \mathrm{pA}, V=-2.5 \mathrm{~V})$, and (c) a $\mathrm{Pc}^{2-}(I=100 \mathrm{pA}, \mathrm{V}=2.5 \mathrm{~V})$ on $3 \mathrm{ML} \mathrm{NaCl} / \mathrm{Ag}(111)$. The spectrum of $\mathrm{HPc}^{-}$ was obtained by resonant energy transfer from a neighboring $\mathrm{ZnPc}$. The vertical dashed lines correspond to TD-DFT calculations of the $\mathrm{S}_{0} \rightarrow \mathrm{S}_{1}$ absorption energies for $\mathrm{H}_{2} \mathrm{Pc}_{1} \mathrm{HPc}^{-}$, and $\mathrm{Pc}^{2-}$, rigidly shifted by $-250 \mathrm{meV}$ to fit the experiment. This shift can be rationalized by considering that the dynamical screening linked to the presence of the $\mathrm{NaCl} / \mathrm{Ag}(111)$ substrate and of the STM tip are not accounted for by our theoretical approach. While this prevents a quantitative comparison between experimental and theoretical energies, this procedure shows that theory accurately predicts the energy shift upon deprotonation. $\mathbf{d}$ Comparison between TD-DFT absorption energies calculated for $\mathrm{H}_{2} \mathrm{Pc}_{1} \mathrm{HPc}^{-}$, and $\mathrm{Pc}^{2-}$ (blue dots) and TD-DFT absorption energies calculated for a $\mathrm{Pc}^{2-}$ as a function of partial positive charges added artificially in its center (white dots). The line through the white dots is a parabolic fit with the parameters given in inset. Source data are provided as a Source Data file. inconsistent with the spectra of Fig. 3. In contrast, our DFT calculations of the deprotonated compounds, $\mathrm{HPc}^{-}$and $\mathrm{Pc}^{2-}$, reveal an unchanged occupancy of the $\pi$-orbitals compared to $\mathrm{H}_{2} \mathrm{Pc}$, explaining why the fluorescence characteristics of these three isoelectronic compounds do not change drastically. The observed blue shifts are well reproduced by the TD-DFT simulations of $\mathrm{HPc}^{-}$and $\mathrm{Pc}^{2-}$ (assuming a systematic shift of the theoretical data to account for the specific environment of the chromophores, see Fig. 3 and "Methods" for details). These blue-shifts may either find their origin in tiny structural reorganisations of the molecule upon deprotonation, or in the electric field generated by the excess $\sigma$-electrons.

To address this issue, we considered the fully symmetric $\mathrm{Pc}^{2-}$ molecule and progressively neutralized it by artificially adding partial positive charges in its center, assuming an unchanged geometry. We then calculated the evolution of the optical gap as a function of the molecular charge (Fig. 3d). These simulations show that the optical gaps of $\mathrm{HPc}^{-}$and $\mathrm{H}_{2} \mathrm{Pc}$ scale extremely well with those of $\mathrm{Pc}^{2-}$ with one and two central positive charges, respectively, eventually demonstrating that the observed shifts are due to the Stark effect generated by the internal charges.

Assuming such an ISE, it may appear surprising to observe a much smaller relative shift $(+20 \mathrm{meV})$ upon removal of the second proton compared to the first deprotonation $(+50 \mathrm{meV})$. This phenomenon is explained by the data of Fig. $3 \mathrm{~d}$, where a parabolic dependency of the optical gap on the central charge is observed, and where the singly and doubly charged chromophores are at either side of the apex of the parabola, leading to very close optical gaps. In the usual case of a chromophore with non-degenerated electronic states placed in a chiefly homogeneous external electrical field, the linear and quadratic Stark effects reflect the respective changes in the permanent dipole moment, $\Delta \vec{\mu}$, and in the polarizability, $\Delta \alpha$, experienced by the ground and excited states of the chromophore ${ }^{25}$. As the $\mathrm{H}_{2} \mathrm{Pc}$ ground and excited states do not exhibit permanent dipole moments $\left(\vec{\mu}_{S_{0}}=\vec{\mu}_{S_{1}}=\overrightarrow{0}\right)$, the presence of a non vanishing linear term (see inset Fig. $3 \mathrm{~d}$ ) may be surprising. This can be elucidated by accounting for the specific geometry of our system where central charges generate a strongly non-homogeneous electric field at the scale of the chromophore, eventually resulting in a linear contribution to the Stark shift of the spectral line (see a detailed perturbation model in Supplementary Note 3). This behavior is therefore characteristic of the close proximity between a chromophore and a point source of electric field. Clearly evidenced in our model system, this "local" effect is inherent to any ISE configuration, including the most complex biological ones, and constitutes the main difference with the usual Stark effect generated by an external electric field.

Vibronic spectra of the deprotonated molecules. The $\mathrm{H}_{2} \mathrm{Pc}$ and $\mathrm{Pc}^{2-}$ spectra also display several vibronic emission lines, similar to tip-enhanced Raman spectra, that can be used as accurate chemical fingerprints of the probed compounds, and which provide detailed information regarding their chemical bond structures ${ }^{40}$. These spectra (Fig. $4 \mathrm{a}$ ) reveal subtle changes of the intensity and energy of several vibronic peaks between $\mathrm{H}_{2} \mathrm{Pc}$ and its doubly deprotonated counterpart. At this stage, however, these changes can indicate either a shift of the mode frequency or the appearance/disappearance of vibronic peaks. In Fig. 4b, we show DFT calculations of the vibronic active modes for the two compounds, which reproduce the experimental frequencies and peak intensities remarkably well. We then use atomic-coordinate displacements provided by DFT to identify the prominent modes of $\mathrm{H}_{2} \mathrm{Pc}$ and of $\mathrm{Pc}^{2-}$ and indicate with black vertical lines in Fig. $4 \mathrm{~b}$ those having a nearly perfect one-to-one correspondence. Experimentally, while the modes below $900 \mathrm{~cm}^{-1}$ all shift to 


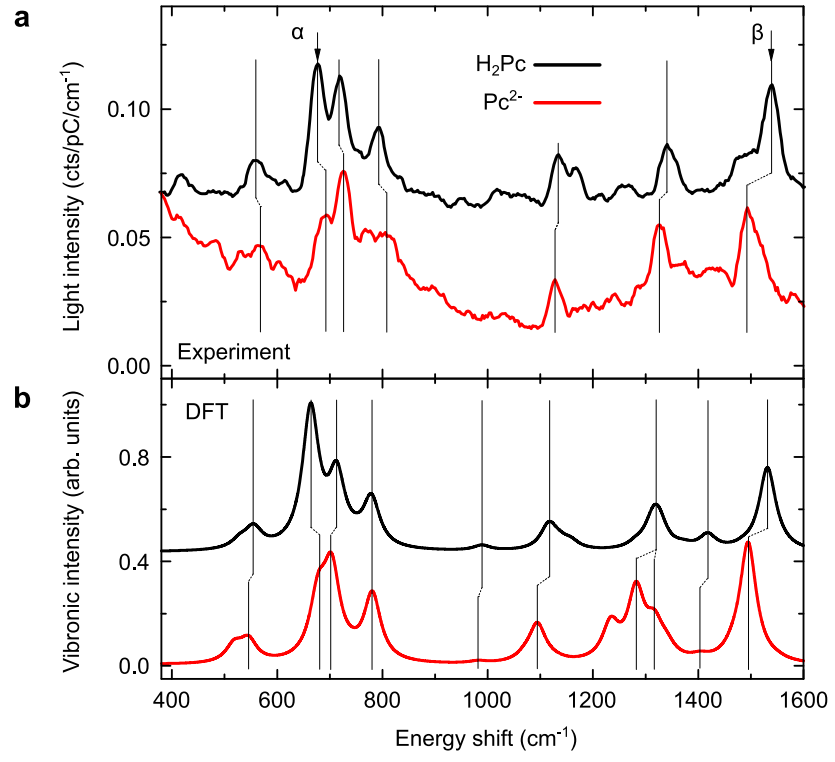

Fig. 4 Effect of the deprotonation on the vibronic signature of the phtahlocyanine molecule. a STML vibronic spectra $(I=100 \mathrm{pA}$, acquisition time $t=120 \mathrm{~s})$ of $\mathrm{H}_{2} \mathrm{Pc}(\mathrm{V}=-2.5 \mathrm{~V})$ and $\mathrm{Pc}^{2-}(\mathrm{V}=2.5 \mathrm{~V})$ molecules on $3 \mathrm{ML}$ of $\mathrm{NaCl}$ compared with (b) theoretical vibronic intensities calculated for the molecules in vacuum. The $\mathrm{Q}_{x}$-line of $\mathrm{H}_{2} \mathrm{Pc}$ and $\mathrm{Q}$-line of $\mathrm{Pc}^{2-}$ are used as the origin of the experimental energy scale. $\alpha$ and $\beta$ indicate vibronic modes for which the four nitrogen atoms of the pyrrole cycles stand nearly still. Source data are provided as a Source Data file.

higher wavenumbers upon double deprotonation of $\mathrm{H}_{2} \mathrm{Pc}$, those above $900 \mathrm{~cm}^{-1}$ display the opposite behavior. Numerically, only one of the low wavenumber modes $(\alpha)$ clearly blue shifts, while the experimental trend in the high wavenumber modes is better accounted for.

Two shifts that are well reproduced by the numerical simulations, both in amplitude and sign, are those of the strongest peak close to $700 \mathrm{~cm}^{-1}(\alpha$ in Fig. 4) and the highest wavenumber mode around $1500 \mathrm{~cm}^{-1}(\beta$ in Fig. 4). For both modes, the four nitrogen atoms of the pyrrole cycles, whether protonated or not, stand nearly still as do the central protons when present (see Supplementary Fig. 10 for the real-space representation of the $\alpha$ and $\beta$ vibrational modes). Hence, their frequency shifts can hardly be explained by an isotopic-like effect associated to deprotonation. In contrast, the two modes entail large motions of the nitrogen atoms bridging the isoindole units and of the carbon atoms they are bound to. The vibronic intensities of these modes are therefore linked to the conjugation paths along the inner rings of $\pi$-orbitals in both species. Their shifts must therefore be attributed to a polarization of the $\pi$ electron system upon removal of the inner proton. To conclude, these two modes may then be seen as undergoing pure vibrational ISE. Most of the other modes entail radial motions of the nitrogen of the isoindoles. Therefore, the respective influence of the vibrational ISE and of isotopic effects induced by the removal of the central protons cannot be disentangled.

\section{Discussion}

Charged states of single molecules have been recently probed in a wide range of experimental schemes involving scanning probe approaches ${ }^{19-23,35,49}$. By simultaneously preserving the $\pi$-orbital structure of $\mathrm{H}_{2} \mathrm{Pc}$ and leaving an excess $\sigma$-electron within the chromophore, the deprotonation procedure reported here provides a unique opportunity to study the Stark effect generated by an internal charge on the fluorescence emission of an individual chromophore. The resulting $\sigma$-type anionic and dianionic molecules constitute model systems allowing us to identify the role played by the proximity between a chromophore and a point-like electrostatic field source. This proximity is responsible for the combined linear and quadratic dependency of the emission energy, a behavior that should occur in any biological systems subject to ISE. It also suggests that chromophores in STML experiments could be used as precise electrostatic sensors of their nanometer-scale environment. Eventually, this work establishes a new biomimetic strategy, based on the control of the local electrostatic environment, to tune and optimise future artificial molecular optoelectronic devices.

\section{Methods}

Experiment. The experiments are performed in ultra-high vacuum at low temperature $(\approx 4.5 \mathrm{~K})$ with an Omicron STM that is combined with an optical set-up adapted to detect light emitted at the STM tip-sample junction. The emitted photons are collected with a lens located on the STM head and then redirected out of the chamber through optical viewports. The light is then focused on an optical fiber coupled to a spectrograph itself connected to a low-noise liquid nitrogencooled CCD camera. The spectral resolution of the setup is better than $1 \mathrm{~nm}$. Further details regarding the optical detection setup can be found in the Supplementary Materials of ref. ${ }^{50}$.

The STM tips are prepared by electrochemical etching of a tungsten wire in a $\mathrm{NaOH}$ solution. The tips are then sputtered with argon ions and annealed under UHV. To optimise their plasmonic response, the tips are eventually indented in clean $\operatorname{Ag}(111)$ to cover them with silver. The $\operatorname{Ag}(111)$ substrate is cleaned by simultaneous argon-ion sputtering and annealing. After this cleaning procedure, $\mathrm{NaCl}$ has evaporated on the $\mathrm{Ag}(111)$ substrate maintained at room temperature. Post-annealing at $\approx 370 \mathrm{~K}$ is performed to induce $\mathrm{NaCl}$ surface reorganization in biand tri-layers. Eventually, the $2-3 \mathrm{ML} \mathrm{NaCl} / \mathrm{Ag}(111)$ sample is introduced into the STM chamber and cooled down to $\approx 4.5 \mathrm{~K}$. $\mathrm{H}_{2} \mathrm{Pc}$ and $\mathrm{ZnPc}$ molecules are then sublimed in very small quantities on the cold sample from a powder located in a quartz crucible. $\mathrm{ZnPc}-\mathrm{HPc}^{-}$molecular dimers on $\mathrm{NaCl}$ are obtained by STM tip manipulation of $\mathrm{ZnPc}$. To this end, the STM tip is first positioned at the edge of a $\mathrm{ZnPc}$ molecule at a bias $V=+2.5 \mathrm{~V}$. In a second step, the tip-molecule distance is slowly reduced until a jump of current occurs, indicating a motion of the molecule. The procedure is reproduced until the desired structure in obtained. The $\mathrm{d} I / \mathrm{d} V$ maps of Fig. 2 in the main paper are recorded in constant current mode (closed feedback loop) with a voltage modulation of $50 \mathrm{mV}$ at a DC voltage of $400 \mathrm{mV}$, whereas the $\mathrm{d} I / \mathrm{d} V$ spectra are recorded in constant height mode (open feedback loop) with a voltage modulation of $20 \mathrm{mV}$. A modulation frequency of $740 \mathrm{~Hz}$ is used for these measurements.

DFT calculation of the ground-state electronic properties. To analyze the molecular transport properties, the electronic structure of the molecule in a vacuum, and the distribution of the corresponding electron charge density in the neutral and charged species, we perform ground-state DFT calculations using the OCTOPUS code ${ }^{51}$. In OCTOPUS the electron density is represented on a realspace grid which does not constrain the localization of the density to predefined atomic orbitals and is therefore suitable to describe the ground-state electron densities of the charged molecules. The electron-ion interaction is modeled in the framework of the pseudopotential approximation. We use the Perdew-Zunger ${ }^{52}$ parametrization of the local density approximation (LDA) correlation and the Slater density functional for the LDA exchange functional ${ }^{53,54}$. We present further analysis of the molecular orbitals, electronic density, and comparison with experimental $\mathrm{d} I / \mathrm{d} V$ images of the molecules with theory in Supplementary Figs. 1-3 and Supplementary Table 1.

Numerical analysis of the ground-state electron densities. We extract from OCTOPUS the ground-state electron densities and calculate the total electrostatic potential $\phi_{\text {tot }}$ generated by the molecular charges to visualize the localization of the excess charge of the deprotonized molecules (as shown in Fig. 2). To that end we solve the Poisson equation:

$$
\Delta \phi_{\text {tot }}=\frac{\rho_{\mathrm{e}}(\mathbf{r})}{\varepsilon_{0}}+\frac{\rho_{\text {ion }}(\mathbf{r})}{\varepsilon_{0}},
$$

with $\varepsilon_{0}$ being the vacuum permittivity. $\rho_{\mathrm{e}}(\mathbf{r})$ is the electron charge density of the valence electrons in the singlet ground state of the molecule and $\rho_{\text {ion }}(\mathbf{r})$ is the charge density of the positive nuclei screened by the core electrons of the respective atoms. We represent the density of the screened nuclei as a sum of Gaussian charge distributions:

$$
\rho_{\text {ion }}(\mathbf{r})=\sum_{i} \frac{Q_{i}}{(2 \pi)^{3 / 2} \sigma_{\text {ion }}^{3}} \exp \left(-\frac{\left|\mathbf{r}-\mathbf{R}_{i}\right|^{2}}{2 \sigma_{\text {ion }}^{2}}\right)
$$


where $Q_{i}$ is the total charge of the screened ion $i$ at position $\mathbf{R}_{i}$. The charge distribution has a width $\sigma_{\text {ion }}=0.05 \mathrm{~nm}$. The electron charge density is extracted from the ground-state DFT calculations in the form of Gaussian cube files. The ground-state electron charge densities for $\mathrm{H}_{2} \mathrm{Pc}_{\mathrm{c}}$ and $\mathrm{HPc}^{-}$are shown in Supplementary Fig. 3c, d, respectively, alongside with the corresponding geometries of the molecules $\left(\mathrm{H}_{2} \mathrm{Pc}\right.$ in Supplementary Fig. 3a and $\mathrm{HPc}^{-}$in Supplementary Fig. 3b). Finally, we solve the Poisson equation [Eq. (1)] numerically on a homogeneously spaced grid by a Fourier-based method.

TD-DFT calculations of the excited-state electronic properties. To address the excited-state properties of the molecules we perform TD-DFT calculations as implemented in the software Gaussian 09 Revision D. $01^{55}$. The TD-DFT calculations (Fig. 3) are carried out with the B3LYP functional and the 6-311G(d,p) basis set. The convergence of 64 roots is asked for when calculating the excited singlet states at the ground state equilibrium geometries of all three compounds which are optimized using the same functional and basis set.

The dependence on a central fractional charge of the transition energy to the first excited singlet state of the doubly deprotonated Pc dianion (Fig. 3d) is calculated in the same way while keeping its equilibrium D4h geometry frozen.

DFT calculation of the geometry of the molecules on $\mathrm{NaCl}$. The geometrical structure of the molecules on $\mathrm{NaCl}$ is relaxed using Quantum Espresso ${ }^{56}$ which is a plane-wave pseudopotential DFT code suitable for the description of the extended substrate. The cubic supercell $(a=53.02457485 \AA)$ included $100 \mathrm{Na}$ and $100 \mathrm{Cl}$ substrate atoms and the calculation is performed at the $\Gamma$ point. The exchange and correlation terms are described using the local density approximation under the approach of Perdew and Zunger ${ }^{52}$. Projector augmented-wave pseudopotentials with core corrections are used to describe the electron-ion interaction ${ }^{57}$. The energy cutoff and density cutoff are set to $50 \mathrm{Ry}$ and $500 \mathrm{Ry}$, respectively. A damped dynamics is used to perform the structural optimization of the system. The atoms are moved according to Newton's equation by using a Verlet algorithm ${ }^{58}$. The structural optimization is stopped when two subsequent total energy evaluations differed by $<10^{-4} \mathrm{Ry}$ and each force component is less than $10^{-3} \mathrm{Ry} / \mathrm{bohr}$.

DFT and TD-DFT calculations of the molecular vibronic properties. Finally, the vibronic intensities (Fig. 4b) are calculated as the square of the sum of the Franck and Condon and Herzberg-Teller amplitudes for each relevant mode independently using Gaussian 09 Revision D.01. To this end, the results of the normal mode calculations are used to define equally spaced discrete distortions spanning the range between the classical turning points of each mode. Repeated TD-DFT calculations of the vertical transition energy and dipole moment to the lowest excited singlet are used to determine the mode displacements and the derivative of the transition dipole moments with respect to the dimensionless normal coordinate through a series of third order polynomial regressions. Since the third order terms are found to be negligible and since the changes in frequencies (from second order terms) between ground and excited states of all the modes that are identifiable in the experimental spectrum are all well below 5\% and would not affect the Franck and Condon overlaps, Duschinsky rotations (mode mixing in the excited state) are ignored. The theoretical vibronic spectra presented are then calculated using the TD-DFT determined intensities by scaling the normal mode frequencies obtained analytically at the DFT level by a factor $0.96^{59}$ and by broadening each line by a Lorentzian $20 \mathrm{~cm}^{-1}$ wide at half maximum.

\section{Data availability}

The data supporting the findings of the present study can be found in Methods or Supplementary Information. All the datasets are also available from the corresponding author upon request. Source data are provided with this paper.

Received: 8 August 2021; Accepted: 7 January 2022;

Published online: 03 February 2022

\section{References}

1. Honig, B. et al. An external point-charge model for wavelength regulation in visual pigments. J. Am. Chem. Soc. 101, 7084-7086 (1979).

2. Gottfried, D., Steffen, M. \& Boxer, S. Large protein-induced dipoles for a symmetric carotenoid in a photosynthetic antenna complex. Science 251, 662-665 (1991).

3. Lockhart, D. \& Kim, P. Internal Stark effect measurement of the electric field at the amino terminus of an alpha helix. Science 257, 947-951 (1992).

4. Drobizhev, M., Tillo, S., Makarov, N. S., Hughes, T. E. \& Rebane, A. Color hues in red fluorescent proteins are due to internal quadratic Stark effect. $J$. Phys. Chem. B 113, 12860-12864 (2009).

5. Boxer, S. G. Stark realities. J. Phys. Chem. B 113, 2972-2983 (2009).
6. Wild, U. P., Güttler, F., Pirotta, M. \& Renn, A. Single molecule spectroscopy Stark effect of pentacene in p-terphenyl. Chem. Phys. Lett. 193, 451-455 (1992)

7. Orrit, M., Bernard, J., Zumbusch, A. \& Personov, R. Stark effect on single molecules in a polymer matrix. Chem. Phys. Lett. 196, 595-600 (1992).

8. Schulte, T. et al. Identification of a single peridinin sensing Chl-a excitation in reconstituted PCP by crystallography and spectroscopy. Proc. Natl. Acad. Sci. USA 106, 20764-20769 (2009).

9. Croce, R. \& van Amerongen, H. Natural strategies for photosynthetic light harvesting. Nat. Chem. Biol. 10, 492 (2014).

10. Kakitani, T., Honig, B. \& Crofts, A. Theoretical studies of the electrochromic response of carotenoids in photosynthetic membranes. Biophys. J. 39, 57 - 63 (1982).

11. Kulzer, F., Matzke, R., Bräuchle, C. \& Basché, T. Nonphotochemical hole burning investigated at the single-molecule level: Stark effect measurements on the original and photoproduct state. J. Phys. Chem. A 103, 2408-2411 (1999).

12. Brunel, C., Tamarat, P., Lounis, B., Woehl, J. C. \& Orrit, M. Stark effect on single molecules of dibenzanthanthrene in a naphthalene crystal and in a n-hexadecane Shpol'skii matrix. J. Phys. Chem. A 103, 2429-2434 (1999).

13. Karotke, S., Lieb, A. \& Hecht, B. Stark-shift microscopy of single emitters. Appl. Phys. Lett. 89, 023106 (2006).

14. Moradi, A., Ristanović, Z., Orrit, M., Deperasińska, I. \& Kozankiewicz, B. Matrix-induced linear Stark effect of single dibenzoterrylene molecules in 2,3dibromonaphthalene crystal. Chem. Phys. Chem. 20, 55-61 (2019).

15. Limot, L., Maroutian, T., Johansson, P. \& Berndt, R. Surface-state Stark shift in a scanning tunneling microscope. Phys. Rev. Lett. 91, 196801 (2003).

16. Fernández-Torrente, I., Kreikemeyer-Lorenzo, D., Stróżecka, A., Franke, K. J. \& Pascual, J. I. Gating the charge state of single molecules by local electric fields. Phys. Rev. Lett. 108, 036801 (2012).

17. Martínez-Blanco, J. et al. Gating a single-molecule transistor with individual atoms. Nat. Phys. 11, 640-644 (2015).

18. Roslawska, A. et al. Mapping Lamb, Stark and Purcell effects at a chromophorepicocavity junction with hyper-resolved fluorescence microscopy. Phys. Rev. X 12, 011012 (2022).

19. Fatayer, S. et al. Reorganization energy upon charging a single molecule on an insulator measured by atomic force microscopy. Nat. Nanotechnol. 13, 376-380 (2018).

20. Patera, L. L., Queck, F., Scheuerer, P. \& Repp, J. Mapping orbital changes upon electron transfer with tunnelling microscopy on insulators. Nature 566, 245-248 (2019).

21. Doppagne, B. et al. Electrofluorochromism at the single-molecule level. Science 361, 251 (2018)

22. Rai, V. et al. Boosting light emission from single hydrogen phthalocyanine molecules by charging. Nano Lett. 20, 7600-7605 (2020).

23. Doležal, J., Canola, S., Merino, P. \& Švec, M. Exciton-trion conversion dynamics in a single molecule. ACS Nano 15, 7694-7699 (2021).

24. Reecht, G., Krane, N., Lotze, C. \& Franke, K. J. $\pi$-radical formation by pyrrolic $\mathrm{H}$ abstraction of phthalocyanine molecules on molybdenum disulfide. ACS Nano 13, 7031-7035 (2019).

25. Bishop, D. M. The vibrational stark effect. J. Chem. Phys. 98, 3179-3184 (1993).

26. Imada, H. et al. Single-molecule investigation of energy dynamics in a coupled plasmon-exciton system. Phys. Rev. Lett. 119, 013901 (2017).

27. Doppagne, B. et al. Single-molecule tautomerization tracking through spaceand time-resolved fluorescence spectroscopy. Nat. Nanotechnol. 15, 207-211 (2020).

28. Liljeroth, P., Repp, J. \& Meyer, G. Current-induced hydrogen tautomerization and conductance switching of naphthalocyanine molecules. Science 317, 1203 (2007).

29. Auwärter, W. et al. A surface-anchored molecular four-level conductance switch based on single proton transfer. Nat. Nanotechnol. 7, 41-46 (2012).

30. Kumagai, T. et al. Controlling intramolecular hydrogen transfer in a porphycene molecule with single atoms or molecules located nearby. Nat. Chem. 6, 41-46 (2014).

31. Kügel, J., Klein, L., Leisegang, M. \& Bode, M. Analyzing and tuning the energetic landscape of $\mathrm{H}_{2} \mathrm{P}_{c}$ tautomerization. J. Phys. Chem. C 121, 28204-28210 (2017)

32. Néel, N., Lattelais, M., Bocquet, M.-L. \& Kröger, J. Depopulation of singlephthalocyanine molecular orbitals upon pyrrolic-hydrogen abstraction on graphene. ACS Nano 10, 2010-2016 (2016).

33. Pham, V. D. et al. Control of molecule-metal interaction by hydrogen manipulation in an organic molecule. J. Phys. Chem. Lett. 7, 1416-1421 (2016).

34. Repp, J., Meyer, G., Olsson, F. E. \& Persson, M. Controlling the charge state of individual gold adatoms. Science 305, 493-495 (2004).

35. Swart, I., Sonnleitner, T. \& Repp, J. Charge state control of molecules reveals modification of the tunneling barrier with intramolecular contrast. Nano Lett. 11, 1580-1584 (2011) 
36. Rabbani, M. G. \& Teraoka, J. Resonance Raman spectra of n-deprotonated $\sigma$ type dianion of porphycenes. Spectrochim. Acta A 76, 207-212 (2010).

37. Imada, H. et al. Real-space investigation of energy transfer in heterogeneous molecular dimers. Nature 538, 364-367 (2016).

38. Chen, G. et al. Spin-triplet-mediated up-conversion and crossover behavior in single-molecule electroluminescence. Phys. Rev. Lett. 122, 177401 (2019).

39. Murray, C. et al. Visible luminescence spectroscopy of free-base and zinc phthalocyanines isolated in cryogenic matrices. Phys. Chem. Chem. Phys. 13, 17543-17554 (2011)

40. Doppagne, B. et al. Vibronic spectroscopy with submolecular resolution from stm-induced electroluminescence. Phys. Rev. Lett. 118, 127401 (2017).

41. Kaiser, K., Gross, L. \& Schulz, F. A single-molecule chemical reaction studied by high-resolution atomic force microscopy and scanning tunneling microscopy induced light emission. ACS Nano 13, 6947-6954 (2019).

42. Cao, S. et al. Energy funneling within multi-chromophore architectures monitored with sub-nanometer resolution. Nat. Chem. 13, 766-770 (2021)

43. Zhang, Y. et al. Visualizing coherent intermolecular dipole-dipole coupling in real space. Nature 531, 623 (2016).

44. Doležal, J. et al. Mechano-optical switching of a single molecule with doublet emission. ACS Nano 14, 8931-8938 (2020).

45. Zhang, Y. Sub-nanometre control of the coherent interaction between a single molecule and a plasmonic nanocavity. Nat. Commun. 8, 15225 (2017).

46. Kuhnke, K. et al. Pentacene excitons in strong electric fields. Chem. Phys. Chem. 19, 277-283 (2018).

47. Yang, B. Sub-nanometre resolution in single-molecule photoluminescence imaging. Nat. Phot. 14, 693-699 (2020).

48. Imada, H. et al. Single-molecule laser nanospectroscopy with micro-electron volt energy resolution. Science 373, 95-98 (2021)

49. Farrukh, A. et al. Bias-polarity dependent electroluminescence from a single platinum phthalocyanine molecule. Chin. J. Chem. Phys. 34, 87-94 (2021).

50. Chong, M. C. et al. Narrow-line single-molecule transducer between electronic circuits and surface plasmons. Phys. Rev. Lett. 116, 036802 (2016).

51. Tancogne-Dejean, N. et al. Octopus, a computational framework for exploring light-driven phenomena and quantum dynamics in extended and finite systems. Phys. Chem. Chem. Phys. 152, 124119 (2020).

52. Perdew, J. P. \& Zunger, A. Self-interaction correction to density-functional approximations for many-electron systems. Phys. Rev. B 23, 5048 (1981).

53. Dirac, P. A. M. Note on exchange phenomena in the Thomas atom. Math. Proc. Camb. Philos. Soc. 26, 376 (1930).

54. Slater, J. A simplification of the Hartree-Fock method. Phys. Rev. 81, 385 (1951).

55. Frisch, M. J. et al. Gaussian 09 Revision D.01Gaussian Inc. (Wallingford CT, 2009).

56. Giannozzi, P. et al. QUANTUM ESPRESSO: a modular and open-source software project for quantum simulations of materials. J. Phys.: Condens. Matter 21, 395502 (2009).

57. Blöchl, P. E. Projector augmented-wave method. Phys. Rev. B 50, 17953-17979 (1994).

58. Verlet, L. Computer "experiments" on classical fluids. i. thermodynamical properties of lennard-jones molecules. Phys. Rev. 159, 98-103 (1967).

59. NIST Computational Chemistry Comparison and Benchmark Database, NIST Standard Reference Database Number 101. http://cccbdb.nist.gov/. Release 21, August 2020, Editor: Russell D. Johnson III.

\section{Acknowledgements}

The authors thank Virginie Speisser and Michelangelo Romeo for technical support. This project has received funding from the European Research Council (ERC) under the European Union's Horizon 2020 research and innovation program (grant agreement No 771850 [G.S.]) and the European Union's Horizon 2020 research and innovation program under the Marie Skłodowska-Curie grant agreement No 894434 [A.R.]. The Labex NIE (Contract No. ANR-11-LABX-0058_NIE [G.S.]), and the International Center for Frontier Research in Chemistry (FRC) [G.S.] are acknowledged for financial support. This work was granted access to the HPC resources of IDRIS under the allocation 2020A0060907459 [H.B.] made by GENCI.

\section{Author contributions}

K.V., B.D., F.S., and G.S. conceived, designed, and performed the experiments. K.V., A.R., and G.S. analyzed the experimental data. T.N., H.B., and A.B. performed the analytical and numerical calculations. All authors discussed the results and contributed to the redaction of the paper.

\section{Competing interests}

The authors declare no competing interests.

\section{Additional information}

Supplementary information The online version contains supplementary material available at https://doi.org/10.1038/s41467-022-28241-8.

Correspondence and requests for materials should be addressed to Guillaume Schull.

Peer review information Nature Communications thanks the anonymous reviewers for their contribution to the peer review of this work.

Reprints and permission information is available at http://www.nature.com/reprints

Publisher's note Springer Nature remains neutral with regard to jurisdictional claims in published maps and institutional affiliations.

Open Access This article is licensed under a Creative Commons Attribution 4.0 International License, which permits use, sharing, adaptation, distribution and reproduction in any medium or format, as long as you give appropriate credit to the original author(s) and the source, provide a link to the Creative Commons license, and indicate if changes were made. The images or other third party material in this article are included in the article's Creative Commons license, unless indicated otherwise in a credit line to the material. If material is not included in the article's Creative Commons license and your intended use is not permitted by statutory regulation or exceeds the permitted use, you will need to obtain permission directly from the copyright holder. To view a copy of this license, visit http://creativecommons.org/ licenses/by/4.0/.

(C) The Author(s) 2022 